\title{
THE OBSERVATIONAL SIGNATURE OF FLUX TUBE WAVES AND AN UPPER LIMIT ON THE ENERGY FLUX TRANSPORTED BY THEM
}

\author{
S.K. SOLANKI and B. ROBERTS \\ Department of Mathematical Sciences \\ University of St Andrews \\ St Andrews, KY16 9SS \\ Scotland
}

\begin{abstract}
The influence of undamped linear longitudinal tube waves on Stokes $V$ profiles is considered. A rough upper limit is set on the energy flux transported by such waves through the photosphere. It is found that this upper limit is larger than the flux in the quiet sun. However, due to the small filling factor of the magnetic elements, the total luminosity of flux tube waves is unlikely to be larger than that of acoustic waves when averaged over the whole sun. Therefore, probably both kinds of waves contribute to chromospheric heating. However, the derived upper limit does not rule out that flux tube waves can significantly enhance the chromospheric brightness in active regions and the supergranular network where the magnetic filling factor is large.
\end{abstract}

\section{Introduction}

Flux tube waves are among the main contenders for transporting the energy required to heat the outer solar atmosphere. However, no observational limits have so far been set on the amount of energy which such waves transport. Due to the small size of the flux tubes (below the best current spatial resolution), no direct observations of locally excited waves exist (but see Giovanelli et al., 1978, for observations of globally excited modes). The indirect evidence is restricted to observations of line broadening (Solanki, 1986) and the modelling of the Stokes $V$ asymmetry (Solanki, 1989). It is, therefore, not possible to directly determine the amount of energy transported by such waves, but we can use the line broadening to set an upper limit. First we briefly describe the general influence of tube waves on the widths of a selected set of spectral lines, since no previous study of this effect exists. We then go on to set a rough upper limit on the energy flux carried by tube waves by comparing with observed line profiles.

\section{Summary of Calculations}

In calculating the waves we follow the theory developed by Roberts and Webb $(1978,1979)$ combined with the following assumptions: 1) The calculations are linear, 2) the thin tube approximation is used, 3) radiative damping is neglected, and 4) the gas within the flux tube is uncoupled from that in its surroundings. A differential equation for the velocity must then be solved, after which the rest of the linear perturbations in atmospheric quantities produced by the wave (e.g. pressure, temperature, density, magnetic field strength) can be derived via the thin tube equations.

The radiative transfer is carried out numerically. For the investigation of basic influences of tube waves on line profiles five hypothetical spectral lines have been chosen. No. 1: Fe I with equivalent width $W_{\lambda}=55$ $\mathrm{m} \AA$ in the quiet sun and excitation potential $\chi_{e}=0 \mathrm{eV}$; No. 2: Fe I, $W_{\lambda}=15 \mathrm{~m} \AA, \chi_{e}=0 \mathrm{eV} ;$ No. 3: $\mathrm{Fe}$ 
I, $W_{\lambda}=110 \mathrm{~m} \AA, \chi_{e}=0 \mathrm{eV}$; No. 4: Fe I, $W_{\lambda}=55 \mathrm{~m} \AA, \chi_{e}=4 \mathrm{eV}$; No. 5: Fe II, $W_{\lambda}=55 \mathrm{~m} \AA, \chi_{e}=3$ $\mathrm{eV}$. All lines have a Landé factor of unity. They have been chosen such that they cover a wide range of $W_{\lambda}$ and $\chi_{e}$. All calculations are for solar disk centre, so that only Stokes $I$ and Stokes $V$ need be considered. We concentrate here on Stokes $V$. Both single- and multi-ray (1.5-D) radiative transfer calculations have been carried out, but the influence of the dimension of the model on the widths of Stokes $V$ profiles is minimal, so that we shall not differentiate between the two in the following.

\section{Influence of Longitudinal Tube Waves on Line Widths}

If we suppress the temperature fluctuations caused by the wave, i.e. consider an isothermal wave, then the widths of all lines are affected similarly. In this case the line width $v_{D}$ can be written as: $v_{D}^{2} \approx\left(\delta \cdot v_{a}(z)\right)^{2}+$ $v_{D_{0}}^{2}$, where $v_{D_{0}}$ is the line width in the absence of the wave, $v_{a}(z)$ is the wave amplitude at the "height of formation", $z$, of the line and $\delta$ is a multiplicative factor which takes into account that the wave profile is closer to a sine wave than to a Gaussian. $\delta$ differs appreciably from unity for practically all waves, suggesting that a Gaussian turbulence broadening model (as used by, for example, Solanki, 1986) will give a different (larger) limit on the flux tube wave energy flux. For waves with sufficiently long wavelengths $\delta$ becomes independent of the line (macroturbulence limit), whereas for short wavelength waves it varies considerably from line to line, depending on the sensitivity of the particular line to microturbulence. Also, for isothermal waves, standing and propagating waves show the same effect on the line width.

If we now let the temperature vary with phase, then standing waves still broaden the lines in the same manner as before (due to the phase difference of $90^{\circ}$ between temperature and velocity fluctuations), while the influence of propagating waves is greatly changed (phase difference of approximately $180^{\circ}$ ). Fig. 1 shows $v_{D}$ vs. $v_{a}(z=0)$ for isothermal (Fig. 1a) and moderately non-isothermal (Fig. 1b) propagating waves with a wavelength of $300 \mathrm{~km}$ and a period of approximately $80 \mathrm{~s}$. Note that the two weaker low excitation $\mathrm{Fe} I$ lines (lines No. 1 and 2) are very strongly affected by the temperature fluctuations, while the others show little or no influence on the line widths. The behaviour of the line widths of the three low excitation lines can be easily understood if we keep their different strengths in mind and follow the weakening and strengthening of the lines in the down- and upflowing phases, respectively. Fig. 1 carries a clear message: as long as we do not know how strong the thermal fluctuations associated with the tube waves are on the sun, it is wise to use a temperature insensitive spectral line for setting a limit on the energy flux transported by such waves.

\section{Upper Limit on the Wave Energy Flux}

The energy flux carried by a tube wave can be written as

$$
F(z)=\left\langle c_{T}(z) \rho(z) v^{2}(z)\right\rangle \approx \frac{1}{2} c_{T}(z) \rho_{0}(z) v_{a}^{2}(z),
$$

where $c_{T}$ is the tube speed (i.e., the propagation speed of a tube wave) and $\rho$ is the gas density ( $\rho_{0}=$ time averaged gas density). We derive the upper limit to $v_{a}$ from a fit to the line widths of Stokes $V$ profiles observed with a Fourier transform spectrometer (FTS) at Kitt Peak. The data have been described by Stenflo et al. (1984). The flux tube model describing the undisturbed atmosphere has been taken from Solanki (1986). In accordance with the conclusion of Sect. 3, we use the Fe II line at $5197.6 \AA$ whose width is unaffected by the thermal fluctuations due to the wave.

From a variety of calculations, using different waves, we arrive at the following preliminary upper limit for the energy flux transported by longitudinal tube waves $F_{\text {Tube }}$ :

$$
F_{\text {Tube }}(5197.6 \AA) \lesssim 3 \times 10^{9} \mathrm{erg} \mathrm{s}^{-1} \mathrm{~cm}^{-2} .
$$

Fe II $5197.6 \AA$ is formed in the lower photosphere, so that $F_{\text {Tube }}$ refers to that height. This limit applies to both an active plage region and an enhanced network region. 


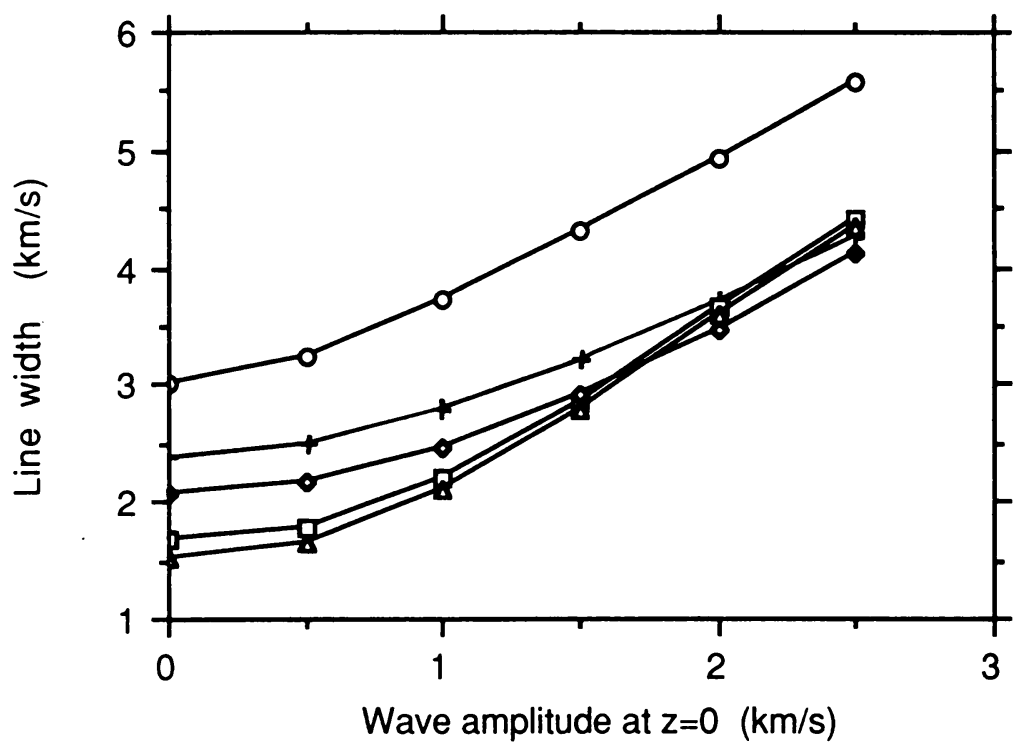

Fig. 1a Line width of Stokes $V$ in $\mathrm{km} \mathrm{s}^{-1}$ vs. the wave amplitude at $z=0$ (corresponding to $\tau_{5000}=1$ in the quiet sun) for isothermal waves with wavelengths of $300 \mathrm{~km}$ and periods of around $80 \mathrm{~s}$. Squares: line 1, triangles: line 2, circles: line 3, diamonds: line 4, plusses: line 5.

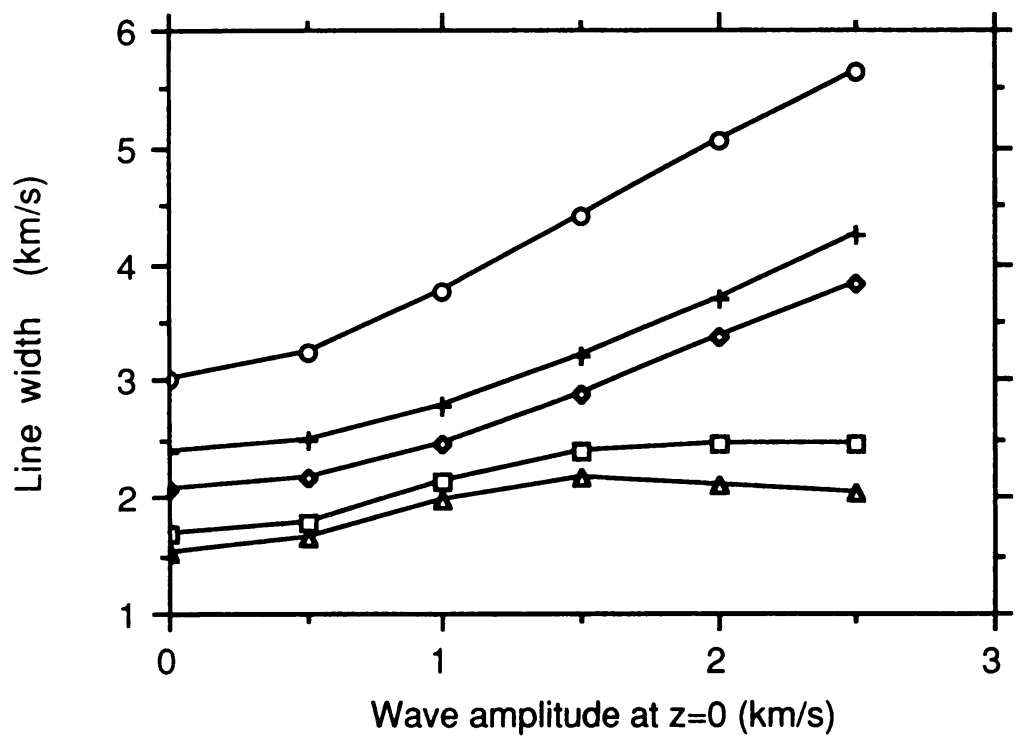

Fig. 1b The same for moderately non-isothermal waves. 
At first sight, the upper limit (2) appears quite large. It is much larger than the recent estimate of Keil and Mosman (1989) for acoustic waves in the quiet sun, who find $F_{\text {Acoustic }} \approx 1 \times 10^{8} \mathrm{erg} \mathrm{s}^{-1} \mathrm{~cm}^{-2}$ after correcting the power upwards by about 2 orders of magnitude to take into account radiative transfer effects. Also, at first sight the upper limit to the tube wave energy flux appears ample to heat the chromosphere, which requires $4 \times 10^{6} \mathrm{erg} \mathrm{s}^{-1} \mathrm{~cm}^{-2}$ in the quiet sun (Withbroe and Noyes, 1977). However, it should be borne in mind that in contrast to the quiet sun values the flux tube values are upper limits. Furthermore, flux tubes cover only a small fraction of the solar surface and, therefore, in order to obtain an "area averaged" value of the limit on the tube wave energy flux it is necessary to multiply the value given in Eq. (2) by the global solar magnetic filling factor. As an optimistic estimate of the global filling factor we take $1 \%$. We are then left with an upper limit of the wave luminosity in flux tubes which is of about the same order as that in the quiet sun, and may be less.

We can reach two main conclusions from the above: 1) Since approximately equal amounts of energy are transported by both kinds of waves, it appears likely that the chromosphere is heated by a combination of tube waves and acoustic waves. Our investigation, therefore, supports the notion of a "basal flux" of acoustic waves which heat the "quiet" chromosphere, first derived from a study of late-type stars by Schrijver (1987). 2) Since magnetic features can cover 10-20\% of the surface area of active regions, flux tube waves potentially carry sufficient energy into the chromosphere above active regions and the network to be primarily responsible for the excess heating seen there (radiative losses are $2 \times 10^{7} \mathrm{erg} \mathrm{s}^{-1} \mathrm{~cm}^{-2}$ in active regions; cf. Withbroe and Noyes, 1977), and so give rise to the "patchiness" of chromospheric Ca II H and K emission seen in spectroheliograms in these lines.

\section{References}

Giovanelli, R.G., Livingston, W.C. and Harvey, J.W. (1978) "Motions in solar magnetic tubes. II. The oscillations", Solar Phys. 59, 49-64.

Keil, S.L. and Mosman, A. (1989) "Observations of High Frequency Waves in the Solar Atmosphere", in Solar and Stellar Granulation, Proc. NATO Advanced Research Workshop, R. Rutten and G. Severino (Eds.), Reidel, Dordrecht, in press

Roberts, B., and Webb, A.R. (1978) "Vertical Motions in an Intense Magnetic Flux Tube.", Solar Phys. 56, 5-35.

Roberts, B., and Webb, A.R. (1979) "Vertical Motions in an Intense Magnetic Flux Tube. III. On the Slender Flux Tube-Approximation", Solar Phys. 64, 77-92.

Schrijver, C.J. (1987) "Magnetic Structure in Cool Stars. XI. Relations Between Radiative Fluxes Measuring Stellar Activity, and Evidence for Two Components in Stellar Chromospheres", Astron. Astrophys. 172, 111-123.

Solanki, S.K. (1986) “Velocities in Solar Magnetic Fluxtubes”, Astron. Astrophys. 168, 311-329.

Solanki, S.K. (1989) “The Origin and Diagnostic Capabilities of the Stokes $V$ Asymmetrie Observed in Solar Faculae and the Network", Astron. Astrophys. in press

Stenflo, J.O., Harvey, J.W., Brault, J.W. and Solanki, S.K. (1984) "Diagnostics of solar magnetic fluxtubes using a Fourier transform spectrometer", Astron. Astrophys. 131, 333-346.

Withbroe, G.L and Noyes, R.W. (1977) "Mass and Energy Flow in the Solar Chromosphere and Corona", Ann. Rev. Astron. Astrophys. 15, 363-387. 